\title{
The Nash-Williams Conjecture and the Dominating Cycle Conjecture
}

\author{
Arthur Hoffmann-Ostenhof * \\ Institute of Information Systems \\ Technical University of Vienna \\ 1040 Vienna, Austria \\ arthur-hoffmann-ostenhof@gmx . at
}

Submitted: Aug 22, 2015; Accepted: Jan 25, 2020; Published: Feb 21, 2020

(c) Arthur Hoffmann-Ostenhof. Released under the CC BY-ND license (International 4.0).

\begin{abstract}
The disproved Nash-Williams conjecture states that every 4-regular 4-connected graph has a hamiltonian cycle. We show that a modification of this conjecture is equivalent to the Dominating Cycle Conjecture.
\end{abstract}

Mathematics Subject Classifications: 05C45

\section{Basic definitions and main result}

For used terminology which is not defined here we refer to [1, 2]. A dominating cycle (DC) of a graph $G$ is a cycle which contains at least one endvertex of every edge of $G$. Let $v \in V(G)$. Then $E_{v}$ denotes the set of edges incident with $v$. A closed trail is a closed walk in which all the edges are distinct. Definitions concerning connectivity parameters are given in the next section. All graphs here are considered to be loopless and finite.

The following two conjectures are well known in graph theory. The first one was disproved by Meredith, see [5].

Nash-Williams Conjecture (NWC): Every 4-regular 4-connected graph has a hamiltonian cycle.

Dominating Cycle Conjecture (DCC): Every cyclically 4-edge connected cubic graph has a dominating cycle.

The DCC is open and so far there is neither a promising approach known to prove it nor to disprove it. For a survey on this conjecture, we refer to [2].

We need the following definitions for introducing the modified NWC.

*Funded by the FWF-Project 26686. 
Definition 1. Let $H$ be a 4-regular graph with a transition system $T$, i.e. $T:=$ $\bigcup_{v \in V(H)}\left\{P_{v}\right\}$ where $P_{v}$ is a partition of the four edges incident with $v$ into two sets of size 2 ; each of these two sets is called a transition of $T$, of $P_{v}$ and of $v$. A trail is said to follow a transition if the two edges of the transition are consecutive edges of the trail. Moreover, $H$ is said to be T-hamiltonian if $H$ contains a $\mathbf{T}$-trail, that is a spanning closed trail $C$ of $H$ such that for each $v \in V(H)$ one of the following two conditions is fulfilled:

a) $\left|E(C) \cap E_{v}\right|=2$ (in this case $C$ may follow no transition of $v$ ).

b) $C$ follows both transitions of $v$ (in this case $\left|E(C) \cap E_{v}\right|=4$ ).

For an example, see Figure 1. Observe that $H$ is $T$-hamiltonian if $H$ is hamiltonian. Hence, $T$-hamiltonicity generalizes the concept of hamiltonian graphs. Now, we introduce the modification of the NWC.
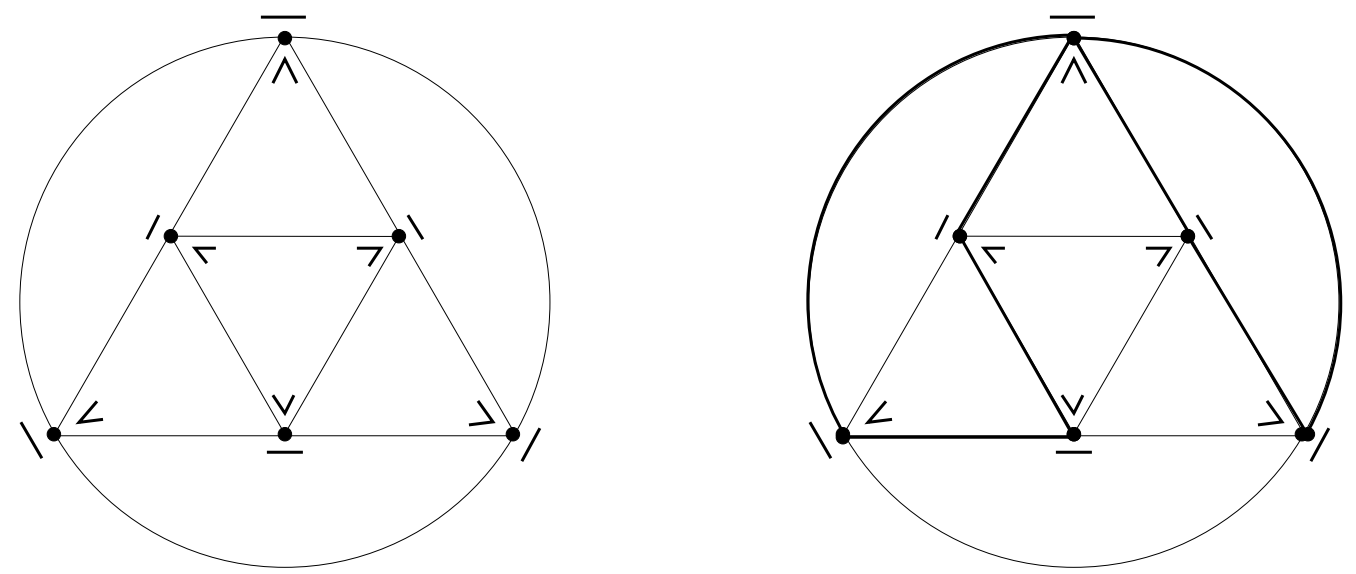

Figure 1: On the left, a graph with a transition system (transitions are illustrated by short bold lines next to those pairs of edges which form a transition), and on the right, a $T$-trail (illustrated in bold) of the same graph with respect to its transition system.

$\boldsymbol{N} \boldsymbol{W} \boldsymbol{C}^{*}$ : Every 4-regular 4-connected graph with a transition system $T$ is $T$-hamiltonian. We state the main result.

Theorem 2. The DCC is equivalent to the $N W C^{*}$.

Theorem 2 implies another result. For stating it we use the following definition.

Definition 3. We say that a cycle dominates a matching $\mathcal{M}$ of a graph $G$ if the cycle contains at least one endvertex of each edge of $\mathcal{M}$. Let $G / \mathcal{M}$ denote the graph which results from $G$ by contracting each edge of $\mathcal{M}$ to a distinct vertex and by deleting all arising loops.

By Corollary 13 of Theorem 2, the subsequent conjecture is equivalent to the DCC.

Conjecture 4. Let $G$ be a cubic graph with a perfect matching $\mathcal{M}$ such that $G / \mathcal{M}$ is 4-connected. Then $G$ contains a cycle which dominates $\mathcal{M}$. 


\section{Proof of the main result}

A graph $G$ is said to be $k$-vertex connected if $|V(G)|>k$ and $G-X$ is connected for every $X \subseteq V(G)$ with $|X|<k$. We abbreviate $k$-vertex connected by $k$-connected. A graph $G$ is called $k$-edge connected if $|V(G)|>1$ and $G-Y$ is connected for every $Y \subseteq E(G)$ with $|Y|<k$. A set $E^{\prime} \subseteq E(G)$ of a connected graph $G$ is called an edge cut of $G$ if $G-E^{\prime}$ is disconnected. Moreover, if at least two components of $G-E^{\prime}$ are not trees, then $E^{\prime}$ is also called a cyclic edge cut of $G$. If $G$ contains two vertex disjoint cycles, then $\lambda_{c}(G)$ is the minimum size over all cyclic edge cuts of $G$ - otherwise we do not define $\lambda_{c}(G)$. Note that every connected cubic graph which does not have two disjoint cycles is either the graph consisting of two vertices and three edges, $K_{4}$, or $K_{3,3}$, see Theorem 1.2 in [4]. Note that within the paper, $\lambda_{c}(G)$ is only considered for cubic graphs $G$. The line graph of a graph $G$ is denoted by $L(G)$. A cycle of length 3 is called a triangle.

For convenience, we split the statement of Theorem 2 into Proposition 5 and Proposition 12 .

Proposition 5. If the $N W C^{*}$ is true, then the DCC is true.

Proof. Let $G$ be a cubic graph satisfying $\lambda_{c}(G) \geqslant 4$. We show that $G$ has a DC. It is well known and not difficult to see that $L(G)$ is 4-regular and 4-connected. Note that every vertex $v \in V(G)$ corresponds to a unique triangle $t_{v}$ of $L(G)$. Moreover, $\left\{E\left(t_{v}\right): v \in V(G)\right\}$ is a partition of $E(L(G))$.

Define the following transitions which imply a transition system $T$ of $L(G)$ : each pair of edges which are incident with the same vertex of $L(G)$ and belong to the triangle $t_{v}$ of $L(G)$ forms a transition, see Figure 2.

Since the NWC* holds, $L(G)$ is $T$-hamiltonian. If $L(G)$ has a hamiltonian cycle, then $G$ has a DC by Theorem 5 in [2] (see also [3]), and we are finished.

Hence, we may assume that $L(G)$ has a $T$-trail $C$ which contains a positive minimum number of 4 -valent vertices. $C$ contains at most one 4 -valent vertex in every triangle $t_{v}$ of $L(G)$. Or else $C$ follows by Definition 1 all transitions in at least two vertices of $t_{v}$. This implies that the remaining third vertex of $t_{v}$ is also a 4 -valent vertex of $C$. Since now $C$ cannot follow both transitions in the third vertex, this is impossible. Let $w$ be a 4-valent vertex of $C$, and let $t_{x}$ with $x \in V(G)$ be one triangle of $L(G)$ which contains $w$. Define the new $T$-trail $C^{\prime}$ of $L(G)$ which results from $C$ by replacing the two edges of $C$ which are contained in $t_{x}$ and incident with $w$ by the remaining edge of $t_{x}$. Then $C^{\prime}$ contains fewer 4-valent vertices than $C$ which contradicts the definition of $C$ and thus finishes the proof.

For the formulation and the proof of the next results we use the following definition.

Definition 6. Let $H$ be a 4-regular graph with a transition system $T$. Let $\mathcal{G}(H, T)$ denote the cubic graph which results from $H$ by firstly splitting every vertex $v$ of $H$ into two vertices $v^{\prime}, v^{\prime \prime}$ of degree 2 such that the corresponding edges of each transition of $T$ remain adjacent and by secondly adding the new edge $e(v):=v^{\prime} v^{\prime \prime}$. Set $M_{T}:=\{e(v): v \in V(H)\}$ which is a perfect matching of $\mathcal{G}(H, T)$ satisfying $\mathcal{G}(H, T) / M_{T} \cong H$. 


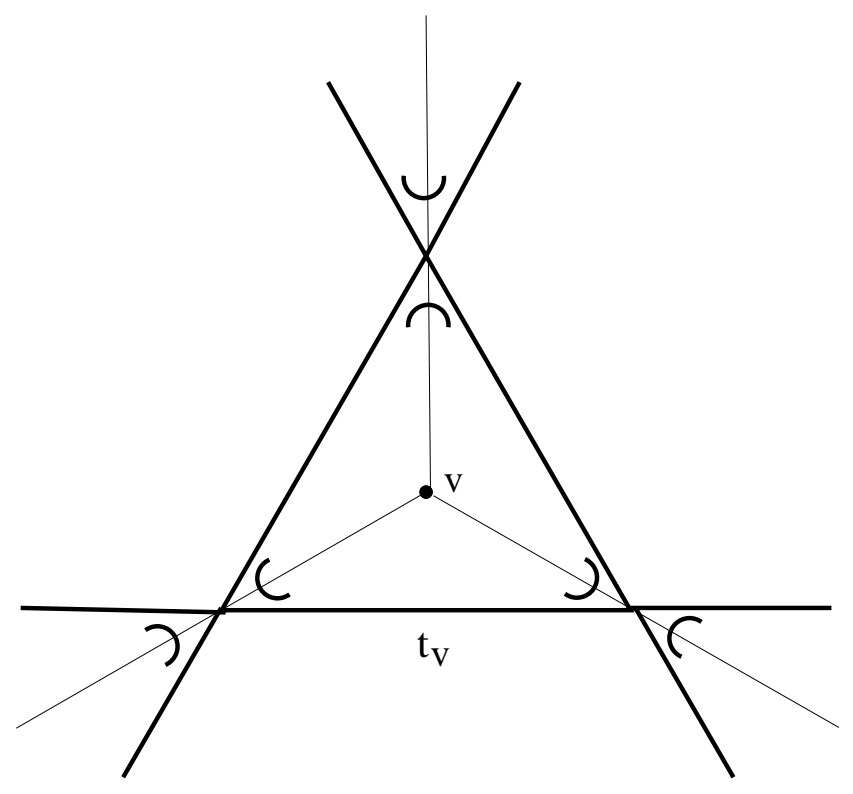

Figure 2: The transitions of the three vertices of $t_{v}$ in the line graph.

The following lemma can be verified straightforwardly.

Lemma 7. Let $H$ be a 4-regular graph with a transition system $T$. Then $\mathcal{G}(H, T)$ has a cycle which dominates $M_{T}$ if and only if $H$ has a T-trail.

We apply the subsequent three lemmata for the proof of the next proposition.

The next lemma is straightforward to prove.

Lemma 8. Let $E_{0}$ be a matching of a cubic graph $G$ such that $G-E_{0}$ consists of two components $X_{1}$ and $X_{2}$. Moreover, let every edge of $E_{0}$ have precisely one endvertex in $X_{i}, i=1,2$. Then $\left|E_{0} \cap M\right|+\left|E_{0}\right|$ is even for every perfect matching $M$ of $G$.

Lemma 9. Let $H$ be a 4-regular 4-connected graph with a transition system $T$. Then $\mathcal{G}(H, T)$ is 3-edge connected. Moreover, if $\mathcal{G}(H, T)$ has a cyclic 3-edge cut $E_{0}$, then one of the two components of $\mathcal{G}(H, T)-E_{0}$ is a triangle.

Proof. Set $G:=\mathcal{G}(H, T)$. Obviously, $G$ is connected. Suppose $G$ has a bridge $f$, then by Lemma 8 (with $E_{0}:=\{f\}$ ), $f \in M_{T}$. Hence, $f$ corresponds to a cut vertex of $H$ which contradicts the assumption that $H$ is 4-connected.

Suppose $E^{\prime}$ is a 2-edge cut of $G$. Then $E^{\prime}$ is a matching, otherwise $G$ has a bridge. By Lemma 8 we have two cases.

Case 1. $E^{\prime} \subseteq M_{T}$.

$H$ is a simple graph, otherwise $H$ is not 4-connected. Thus, $G$ is also a simple graph. Therefore both components of $G-E^{\prime}$ contain more than two vertices. Hence, $E^{\prime}$ corresponds to a vertex 2-cut of $H$ which contradicts the definition of $H$.

Case 2. $M_{T} \cap E^{\prime}=\emptyset$.

Then $E^{\prime}$ corresponds to a 2-edge cut of $H$. Since $H$ is 4-connected and since the edge connectivity is greater than or equal to the vertex connectivity, this is impossible. 
Hence, $G$ is 3-edge connected which finishes the first part of the proof.

Let $E_{0}$ be a cyclic 3 -edge cut of $G$. Then $E_{0}$ is a matching and $G-E_{0}$ consists of two components, otherwise $G$ would contain a $k$-edge cut for some $k \in\{1,2\}$. Moreover, at most one component of $G-E_{0}$ is a triangle, since otherwise $|V(H)|=3$ and $H$ is not 4-connected.

Suppose by contradiction that no component of $G-E_{0}$ is a triangle. Hence both components have more than three vertices. Since every graph contains an even number of vertices of odd degree, every component has at least five vertices. Denote one of the two components of $G-E_{0}$ by $L$. By Lemma 8 and by reasons of symmetry, we need to consider two cases: Case A and Case B. Set $E_{0}=\left\{e_{1}, e_{2}, e_{3}\right\}$.

Case A. $M_{T} \cap E_{0}=\left\{e_{1}\right\}$.

Let us suppose first that $|V(L)|>5$.

Set $E^{*}:=\left\{e \in E(L) \cap M_{T}: e\right.$ is incident with a 2-valent vertex of $\left.\mathrm{L}\right\}$. Obviously, $1 \leqslant\left|E^{*}\right| \leqslant 2$ (depending on whether one edge of $M_{T} \cap E(L)$ covers one endvertex of $e_{2}$ and one of $\left.e_{3}\right)$. $E^{*} \cup\left\{e_{1}\right\}$ corresponds to a vertex subset of $V(H)$ which we denote by $W$. Since $|V(L)|>5$, it follows that $H-W$ is not connected. Moreover, $W$ is a $j$-vertex cut of $H$ for some $j \in\{2,3\}$ which contradicts the assumption that $H$ is 4-connected.

Thus, we may assume that $|V(L)|=5$.

Hence, $\left|M_{T} \cap E(L)\right|=2$. Set $\{a, b\}:=M_{T} \cap E(L)$. Since $H$ is a simple graph, $a$ and $b$ have the following properties:

(1) $a$ and $b$ are not contained together in a cycle of length 4 .

(2) Neither $a$ nor $b$ is contained in a triangle.

Denote by $v_{1}$ the unique 2-valent vertex of $L$ which is neither matched by $a$ nor by $b$. Denote by $a_{1}$ and $b_{1}$ the remaining two 2-valent vertices in $L$. If $a_{1} b_{1} \in E(L)$, then $a_{1} b_{1} \notin$ $M_{T}$; otherwise the two vertices in $H$ corresponding to $e_{1}$ and $a_{1} b_{1}$ would form a vertex 2 -cut of $H$. Hence we can set $a:=a_{1} a_{2}$ and $b:=b_{1} b_{2}$ such that $V(L)=\left\{v_{1}, a_{1}, a_{2}, b_{1}, b_{2}\right\}$.

By (2), $v_{1}$ is adjacent to precisely one endvertex of $a$ and to one of $b$. Thus, and by reasons of symmetry we have three possibilities:

Subcase 1. $\left\{v_{1} a_{1}, v_{1} b_{1}\right\} \subseteq E(L)$.

Then $G$ must contain the edge $a_{2} b_{2}$ twice which is impossible since $G$ is a simple cubic graph.

Subcase 2. $\left\{v_{1} a_{1}, v_{1} b_{2}\right\} \subseteq E(L)$.

Then $L$ contains the triangle consisting of the vertices $b_{1}, b_{2}, a_{2}$ which contradicts (2).

Subcase 3. $\left\{v_{1} a_{2}, v_{1} b_{2}\right\} \subseteq E(L)$.

Then $L$ contains either double edges or a cycle of length 4 consisting of the vertices $a_{1}, a_{2}, b_{1}, b_{2}$ which contradicts (1).

Hence, Case A cannot occur.

Case B. $\left|M_{T} \cap E_{0}\right|=3$.

Since both components of $G-E_{0}$ have at least five vertices and since $E_{0} \subseteq M_{T}, E_{0}$ corresponds to a vertex 3 -cut of $H$ which contradicts the definition of $H$ and thus finishes the proof.

Definition 10. Let $H$ be a 4-regular simple graph with a transition system $T$. Denote by $\mathcal{G}^{\prime}(H, T)$ the graph which results from $\mathcal{G}(H, T)$ by contracting each triangle into a vertex. 
Note that each pair of triangles of $\mathcal{G}(H, T)$ (in Definition 10) is vertex disjoint. Hence, $\mathcal{G}^{\prime}(H, T)$ is well defined and cubic.

Lemma 11. Let $H$ be a 4-regular 4-connected graph with a transition system $T$. Then either $\mathcal{G}^{\prime}(H, T) \in\left\{K_{4}, K_{3,3}\right\}$ or $\lambda_{c}\left(\mathcal{G}^{\prime}(H, T)\right) \geqslant 4$.

Proof. Since $|V(H)| \geqslant 5,|V(\mathcal{G}(H, T))| \geqslant 10$ and thus $\left|V\left(\mathcal{G}^{\prime}(H, T)\right)\right| \geqslant 4$. Suppose first that $\mathcal{G}^{\prime}(H, T)$ does not contain two disjoint cycles. Since $\left|V\left(\mathcal{G}^{\prime}(H, T)\right)\right| \geqslant 4$ and by Theorem 1.2 in [4], it follows that $\mathcal{G}^{\prime}(H, T) \in\left\{K_{4}, K_{3,3}\right\}$.

Now, assume that $\mathcal{G}^{\prime}(H, T)$ has two disjoint cycles. Suppose by contradiction that $E_{0}^{\prime}$ is a cyclic $k$-edge cut of $\mathcal{G}^{\prime}(H, T)$ for some $k \in\{1,2,3\}$. Then $E_{0}^{\prime}$ corresponds to a cyclic $k$-edge cut $E_{0}$ of $\mathcal{G}(H, T)$. Lemma 9 implies that $k=3$ and that one of the two components of $\mathcal{G}(H, T)-E_{0}$ is a triangle. Since this triangle is contracted to a vertex in $\mathcal{G}^{\prime}(H, T)$, one of the two components of $\mathcal{G}^{\prime}(H, T)-E_{0}^{\prime}$ is a vertex. Hence, $E_{0}^{\prime}$ is not a cyclic edge cut which is a contradiction and thus finishes the proof.

Proposition 12. If the DCC is true, then the $N W C^{*}$ is true.

Proof. Let $H$ be a 4-regular 4-connected graph with a transition system $T$. Since $H$ is 4-connected, $|V(H)| \geqslant 5$. Set $G:=\mathcal{G}(H, T)$. Then $|V(G)| \geqslant 10$ and we have the following two cases.

Case 1. $\lambda_{c}(G) \geqslant 4$. Then, by assumption $G$ has a DC which thus dominates $M_{T}$ (Definition 6). By Lemma 7, $H$ is $T$-hamiltonian.

Case 2. $\lambda_{c}(G)<4$. Consider $\mathcal{G}^{\prime}(H, T)$ (see Definition 10). Every edge of $\mathcal{G}^{\prime}(H, T)$ corresponds to an edge of

$$
E(G)-\{e \in E(G): e \text { is contained in a triangle of } G\} .
$$

Thus, every subgraph $X^{\prime}$, say, of $\mathcal{G}^{\prime}(H, T)$ induces by its corresponding edge set in $G$, a subgraph of $G$ which we denote by $X$.

Note that $K_{4}$ and $K_{3,3}$ have a dominating cycle. Therefore, by Lemma 11 and since the DCC holds, $\mathcal{G}^{\prime}(H, T)$ has a dominating cycle $C^{\prime}$. The corresponding subgraph $C \subseteq G$ is not a cycle if and only if there is a vertex $v^{\prime} \in V\left(C^{\prime}\right)$ which has been obtained by contracting a triangle in $G$. We denote this triangle by $\triangle\left(v^{\prime}\right)$ and call such a vertex $v^{\prime}$, a bad vertex of $C^{\prime}$. We define the cycle $\widetilde{C} \subseteq G$ depending on $C^{\prime}$ : for each bad vertex $v^{\prime}$ of $C^{\prime}$, we extend $C \subseteq G$ to $\widetilde{C}$ by adding the unique path of length 2 which is contained in $\triangle\left(v^{\prime}\right)$ and which connects two endvertices of two edges of $C$; if $C^{\prime}$ has no bad vertex, then $C$ is already a cycle and we set $\widetilde{C}:=C$. We show that $\widetilde{C}$ dominates $M_{T}$.

Since $H$ is 4-connected, $H$ is simple. Thus, no triangle of $G$ contains an edge of $M_{T}$. Hence, it suffices to show that the edge $e \in E(G)$ is dominated by $\widetilde{C}$ for every $e^{\prime} \in E\left(\mathcal{G}^{\prime}(H, T)\right)$. If $e^{\prime} \in E\left(C^{\prime}\right)$, then $e \in E(C)$ and thus $e$ is dominated by $\widetilde{C}$. If $e^{\prime} \notin E\left(C^{\prime}\right)$, then the following two cases are possible. Firstly, if $e^{\prime}$ is a chord of $C^{\prime}$, then by the construction of $\widetilde{C}$ both endvertices of $e$ are contained in $\widetilde{C}$. Secondly, if precisely one endvertex of $e^{\prime}$ is contained in $C^{\prime}$, then one endvertex of $e$ is contained in $\widetilde{C}$.

Hence, $\widetilde{C}$ dominates $M_{T}$. By Lemma $7, H$ is $T$-hamiltonian which finishes the proof. 
Now, Theorem 2 follows easily from Proposition 5 and Proposition 12.

Corollary 13. Conjecture 4 is equivalent to the DCC.

Proof. Suppose that Conjecture 4 holds. We show that in this case the NWC* holds which implies by Theorem 2 the truth of the DCC. Let $H$ be a 4-regular 4-connected graph with a transition system $T$. Since Conjecture 4 holds, the cubic graph $\mathcal{G}(H, T)$ has a cycle dominating $M_{T}$ and thus by Lemma 7, the first part of the proof is finished.

Suppose that the DCC and thus by Theorem 2 also the NWC* holds. Let $G$ and $\mathcal{M}$ be given as in Conjecture 4. We want to find a cycle which dominates $\mathcal{M}$. Set $H:=G / \mathcal{M}$ and let $T$ be the transition system of $H$ such that $\mathcal{G}(H, T) \cong G$. Since the NWC* holds, $H$ is $T$-hamiltonian. By Lemma $7, G$ has a cycle dominating $M_{T}$ where $M_{T}$ equals $\mathcal{M}$ which finishes the proof.

\section{References}

[1] J.A. Bondy, U.S.R. Murty. Graph Theory, Springer 2008.

[2] H.J. Broersma, Z. Ryjáček, P. Vrána. How many conjectures can you stand: a survey. Graphs Combin. 28 (2012), 57-75.

[3] F. Harary, C.St.J.A. Nash-Williams. On eulerian and hamiltonian graphs and line graphs. Can. Math. Bull. 8 (1965), 701-710.

[4] W. McCuaig. Edge reductions in cyclically $k$-connected cubic graphs. J. Combin. Theory Ser. B 56 (1992), 16-44.

[5] G.H.J. Meredith. Regular $n$-valent $n$-connected nonhamiltonian non- $n$-edge-colorable graphs. J. Combin. Theory Ser. B 14 (1973), 55-60. 